new patients. However, these sites do not provide information on malpractice cases or disciplinary actions.

Some popular medical resources aimed at patients:

Yahoo! Health http://health.yahoo.com

WebMD http://www.webmd.com

Dr. Koop's Web Page http://www.drkoop.com

American Hospital Directory

http://www.ahd.com

D. John Doyle MD PHD FRCPC

Toronto, Ontario

\title{
Review of Airway Cam Video Series Volume 3: Advanced Airway Imaging and Laryngoscopy Techniques
}

28 min; $\$ 150$ (US); written and produced by Richard M. Levitan, E. Andrew Ochroch, 2000.

VHS NTSC format. www.airwaycam.com

This is the third in an award-winning series of educational videos by Levitan demonstrating the value of the Airway $\mathrm{Cam}^{\mathrm{TM}}$ as a teaching device. It begins with historical information about laryngoscopy and progresses to a demonstration of the airway anatomy as the endoscopist navigates a fibreoptic nasopharyngoscope beyond his own turbinates toward his arytenoids while narrating the video. Following this, we view the larynx through a stroboscopic telescope for a more detailed dynamic view of the anatomy. The footage and commentary are excellent, though the endoscopist's singing leaves something to be desired.

The video then demonstrates a variety of straight and curved blades, with a brief discussion of the advantages and demonstration of the specific techniques each requires. Here the video goes well beyond descriptions found in texts and other videos. The Airway $\mathrm{Cam}^{\mathrm{TM}}$ is a head-mounted video camera, which offers a live parallax-free view as seen by the laryngoscopist. The producers demonstrate a variety of laryngoscopic views obtained with Miller, Wisconsin, Guedell, Macintosh and McCoy blades employing external laryngeal manipulation when required.

This video has something for everyone. It will satisfy the medical student who is uncertain about what he should be looking for and how best to accomplish this. It will also satisfy the airway expert with its clear commentary and teaching pearls. I think that it has a place in the video library of every anesthesia department.

Richard M. Cooper MD FRCPC

Toronto, Ontario 